\title{
Coronavirus. un nome comune (di virus) per una malattia non comune
}

Sara Giovine

PUBBLICATO: 31 MARZO 2020

L

a parola coronavirus, divenuta ormai di uso comune anche nella lingua corrente in seguito al diffondersi nel nostro paese (e nel mondo) dell'epidemia di COVID-rg, nasce come tecnicismo della biologia, nel significato di 'qualsiasi virus appartenente alla famiglia omonima di virus dall'aspetto simile a una corona, causa di malattie del sistema respiratorio nell'uomo e del sistema respiratorio e gastrointestinale negli animali'. Come tale la forma è infatti registrata dai principali dizionari italiani, tra cui il Grande dizionario italiano dell'uso di De Mauro, che per primo registra la voce, includendola nel supplemento del 2003 - con datazione a questo stesso anno - dedicato alle Nuove parole italiane dell'uso. Come giustamente glossato nell'opera di De Mauro, il termine rappresenta un prestito integrale dall'inglese coronavirus, una parola composta a partire dal sostantivo latino corona 'corona, aureola' (con riferimento all'aspetto del virus, caratterizzato da un involucro sferico esterno con strutture glicoproteiche disposte a corona sulla superficie) e dal termine del latino scientifico virus 'virus' (per la cui storia ed evoluzione semantica a partire dal latino classico si rimanda al tema del mese del marzo 2020 di Claudio Marazzini, In margine a unépidemia: risvolti linguistici di un virus).

Il termine è stato infatti coniato in ambito anglosassone alla metà degli anni Sessanta, in seguito alla scoperta di un gruppo di ricercatori della Common Cold Research Unit (attiva in un ospedale militare nei pressi di Salisbury, in Inghilterra), che individuò per la prima volta questa tipologia di virus nelle cavità nasali dei pazienti con raffreddore comune: al virus, dopo ulteriori studi e dopo l'osservazione al microscopio, che ne ha rivelato il caratteristico aspetto superficiale a corona, è stato quindi attribuito il nome di coronavirus (come è stato possibile ricostruire grazie alla consultazione di alcuni articoli specialistici dedicati alla storia del virus). La voce è registrata come tecnicismo della medicina dai principali strumenti lessicografici inglesi, tra cui il Cambridge Dictionary e l'Oxford English Dictionary, che riporta come data di prima attestazione il 1968 , in un articolo della rivista scientifica "Nature", in cui viene appunto data comunicazione del nome del virus:

In the opinion of the eight virologists, these viruses are members of a previously unrecognised group which they suggest should be called the coronaviruses, to recall the characteristic appearance by which these viruses are identified in the electron microscope. [Secondo gli otto virologi, questi virus sono membri di un gruppo precedentemente non riconosciuto che essi suggeriscono di chiamare coronavirus, con riferimento al caratteristico aspetto con il quale sono stati identificati al microscopio elettronico]. (Virology: Coronaviruses, "Nature", sez. News and Views, vol. 220, p. 650, I6/1I/1968)

In italiano, la prima occorrenza della parola che si è potuta rinvenire attraverso l'interrogazione di Google Libri e degli archivi dei principali quotidiani nazionali è di qualche anno successiva, e risale più precisamente al luglio del I970, in un articolo della "Stampa" (probabilmente tradotto dall'inglese), scritto dal virologo Arie Zuckerman. In esso lo scienziato inglese dà conto delle sue recenti scoperte sull'epatite virale, relative in particolare all'identificazione di un ceppo di coronavirus in uno dei soggetti malati studiati:

Zuckerman, Taylor e Almeida, in una comunicazione preliminare del I969, descrivevano 
l'identificazione di un ceppo di coronavirus nel siero di un soggetto con epatite attiva cronica e cirrosi. [...] Il virus appartiene allo stesso gruppo dei coronavirus che è causa riconosciuta dell'epatite del topo. (Arie Zuckerman, Moderni studi sull'epatite virale epidemica e da siero, "la Stampa", 30/7/197o, p. I4).

La scoperta viene riportata anche in alcune pubblicazioni italiane di taglio specialistico dello stesso anno, che sono probabilmente precedenti all'articolo divulgativo della "Stampa" (è infatti plausibile che la scoperta sia stata prima resa nota alla comunità scientifica, in pubblicazioni di argomento medico, e poi divulgata al resto della popolazione), ma di cui non conosciamo tuttavia il mese esatto di pubblicazione:

In un caso di epatite cronica attiva, negativo per l'antigene Australia, è stata osservata al microscopio elettronico (Zuckermann et al.) la presenza di particelle virus-simili, diverse morfologicamente da quelle dell'antigene Australia, ma che richiamavano l'aspetto dei Coronavirus [...]. (Rapporti tra Coronavirus e epatite virale, "Annali della Sanità pubblica", vol. XXXI, I970, p. 9).

Proprio recentemente un altro tipo di virus è entrato in lizza pretendendo la paternità dell'epatite infettiva, il coronavirus, vicino per alcuni aspetti al virus $\left(\mathrm{MHV}_{3}\right)$ che provoca l'epatite nel topo. Zuckerman e coll. hanno reperito particelle riportabili al virus in questione nel siero di un soggetto affetto da epatite cronica attiva [...]. (Aggiornamenti su malattie infettive ed immunologia, vol. XVI, 1970, p. 9)

Nei decenni successivi, la diffusione della parola resta circoscritta agli ambiti specialistici di origine, della biologia e della medicina, comparendo quasi esclusivamente in pubblicazioni di argomento medico o in riviste di zootecnia e veterinaria che approfondiscono le patologie causate dal virus anche negli animali. Tra gli anni Ottanta e Novanta se ne rinvengono tuttavia attestazioni sporadiche anche nelle maggiori testate nazionali, per lo più in coincidenza dei mesi invernali, in articoli sull'influenza e altre malattie stagionali, che citano il virus tra i principali responsabili del raffreddore e di altre sindromi parainfluenzali. Per esempio:

Sono gli altri virus respiratori - "rinovirus" (già loro sono più di roo), "adenovirus", "parainfluenzali", "coronavirus", etc. - che nel frattempo, in America come da noi, fanno un po' quello che vogliono, in un intreccio di sgradevoli e pressoché identici effetti similinfluenzali. (Ezio Minetto, Linfluenza, per ora, tace. Comandano gli altri virus, "la Stampa", 6/2/1983, p. 8)

Il raffreddore - spiega Gwaltney - è una malattia infettiva, che può essere causata da vari tipi di virus. Nel 50 per cento dei casi, però, i responsabili sono i rinovirus (appunto i virus del naso), ed è su questi ultimi che si è concentrata la maggiore attenzione. Più raramente sono in gioco i coronavirus, gli adenovirus, i virus parainfluenzali e altri ancora. (Pietro Dri, Raffreddore arrenditil, "Corriere Salute", 24/6/199I, p. 20)

È solo nel corso del 2003 che si assiste a un primo, parziale ingresso della forma anche nella lingua comune, come conseguenza dell'ampia diffusione di articoli e notizie relativi all'epidemia di polmonite atipica, meglio nota come SARS (acronimo del nome scientifico inglese Severe Acute Respiratory Syndrome 'sindrome respiratoria acuta grave'), scoppiata in Cina nel novembre del 2002 e poi diffusasi in altri paesi asiatici, in Canada e, in misura minore, anche in altri stati del mondo, che gli scienziati rivelano essere causata proprio da un coronavirus, differente da quello del raffreddore comune e mai individuato prima nell'uomo. Nei principali media italiani si comincia a parlare della malattia, e quindi del virus, dalla fine di marzo, in seguito all'allerta globale lanciata dall'Organizzazione mondiale della Sanità e all'attivazione dei primi controlli negli aeroporti internazionali: tra la primavera e l'estate del 2003 si moltiplicano, in rete e nei giornali, articoli e interventi che trattano l'argomento da diversi punti di vista, per lo più menzionando anche il virus responsabile dell'epidemia e contribuendo così a 
un sensibile incremento d'uso della parola:

L'agente infettivo della Sars non è ancora stato definitivamente identificato. [...] Ieri ricercatori dell'università di Hong Kong hanno annunciato di avere identificato, al microscopio elettronico, un nuovo ceppo virale, prima sconosciuto, che rientra nella famiglia dei coronavirus, responsabili anche dei raffreddori comuni. (Adriana Bazzi, Polmonite, controlli in tutti gli aeroporti, "Corriere della Sera", sez. Cronache, $28 / 3 / 2003$, p. 22).

Il virus responsabile della polmonite anomala sembra essere un coronavirus, della stessa famiglia degli agenti che causano i comuni raffreddori. (s.n., Polmonite killer, ecco sintomi e cure, Repubblica.it, sez. Cronaca, $\mathrm{I} / 4 / 2003)$

Anche dopo l'attribuzione al virus del nome scientifico di SARS-CoV (acronimo dell'inglese Severe Acute Respiratory Syndrome Coronavirus), in rete e nei giornali questo continua a essere indicato per lo più come "il nuovo coronavirus", o "il coronavirus della polmonite atipica (o della Sars)", e talora anche più semplicemente come "il coronavirus". La parola comincia inoltre a comparire anche nelle varianti minoritarie, con grafia analitica corona virus e corona-virus; in quella "italianizzata", con collocazione a sinistra della testa semantica del composto, virus corona; e infine anche in quella ridotta, di sapore più colloquiale corona, che sono forse impiegate dai giornalisti per evitare il ripetersi a breve distanza della stessa forma. Per esempio:

I virus corona, cosi detti a causa della loro tipica forma elicoidale, sono una famiglia di agenti patogeni scoperta abbastanza di recente, e non molto conosciuta, che può infettare anche numerosi mammiferi e uccelli. [...] La capacità dei corona virus di infettare sia gli animali che gli esseri umani, sottolineano i ricercatori cinesi, fa pensare che il ceppo letale sia una mutazione di un virus animale [...]. (Claudia Di Giorgio, Oms: controlli su tutti i viaggiatori dall'Asia, Repubblica.it, sez. Cronaca, 27/3/2003)

Ieri la conferma dell'Organizzazione mondiale della sanità (Oms): I3 laboratori in dieci Paesi sono giunti alla stessa conclusione, dopo aver sottoposto il «corona» alla prova dei postulati di Koch. (Daniela Daniele, Trovato il colpevole della polmonite fulminante, "la Stampa", sez. Cronache, 17/4/2003, p. I2)

Con l'esaurirsi dell'epidemia di SARS nell'estate del 2003, si assiste al progressivo diradarsi delle occorrenze della parola, il cui uso al di fuori degli ambiti specialistici nei mesi successivi e per tutto il successivo decennio si riduce nuovamente ad attestazioni sporadiche, riscontrabili quasi esclusivamente in articoli divulgativi sui virus responsabili delle sindromi simil influenzali. A una rinnovata fortuna della voce contribuisce l'esplodere, tra la fine del 2012 e l'estate del 20I3, di una nuova epidemia di polmonite in alcuni paesi del Medioriente, inizialmente indicata come "Nuova Sars" e poi ufficialmente denominata MERS (acronimo dell'inglese Middle East Respiratory Syndrome 'sindrome respiratoria mediorientale'), anch'essa causata da un coronavirus, simile a quello della SARS ma da esso distinto, a cui viene successivamente dato il nome di MERS-CoV (Middle East Respiratory Syndrome Coronavirus). In Italia le notizie sulla malattia cominciano a circolare dal maggio del 20I3, in seguito al verificarsi dei primi casi in Europa e alle raccomandazioni dell'Oms per la prevenzione della diffusione dell'epidemia; come già avvenuto per la SARS, nella lingua dei giornali al nome scientifico del virus si tende spesso a preferire la più semplice indicazione di "nuovo coronavirus" o di "coronavirus (della) Mers", o in alternativa di "coronavirus del Medio Oriente":

L'Organizzazione Mondiale della Sanità, per voce del Direttore generale, Margaret Chan, ha espresso tutta la sua preoccupazione per la diffusione rapida che sta mostrando il nuovo coronavirus, senza che vi sia stata una reazione adeguata da parte dei Paesi mondiali. (s.n., La Sars ora minaccia tutti, laStampa.it, sez. Salute, 30/5/2013) 
I laboratori di virologia se l'aspettavano. Esiste in Italia la rete di centri Influnet, che normalmente controlla l'influenza di stagione. Questa volta però ci si era preparati anche per l'eventuale arrivo del coronavirus Mers. (ed., Il virus si trasmette meno dell'influenza ma l'infezione va fermata sul nascere, "la Repubblica", sez. Cronaca, I/6/2013, p. I8)

Tra il 2014 e il 2015 si riscontra ancora qualche occorrenza della parola, in articoli di cronaca che danno notizia degli ultimi casi della malattia, mentre negli anni successivi si ritorna a un impiego del tutto sporadico della forma, con attestazioni isolate nella lingua dei giornali. Questo fino al gennaio del 2020, quando i media italiani cominciano a parlare della nuova epidemia di polmonite scoppiata nel dicembre dell'anno precedente a Wuhan (nella provincia cinese centrale dello Hubei), causata da un nuovo tipo di coronavirus, che viene identificato dai ricercatori cinesi proprio nei primi giorni del 2020:

Gli scienziati cinesi hanno identificato un nuovo tipo di coronavirus dietro all'epidemia di polmonite diffusasi a Wuhan, nella provincia centrale cinese dello Hubei, a metà dicembre scorso e che ha finora colpito 59 persone. (Il virus che fa paura all'Oriente: epidemia di polmonite alla vigilia dellesodo per il Capodanno cinese, laStampa.it, sez. Salute, 9/I/2020)

Nelle settimane successive, con il verificarsi dei primi casi anche al di fuori della Cina e soprattutto con la diffusione della notizia dei due turisti cinesi in vacanza a Roma risultati positivi al virus, si moltiplicano in rete e nei giornali le occorrenze della parola, che subiscono unulteriore crescita a partire dall'ultima settimana di febbraio, in seguito al contagio dei primi pazienti italiani e all'individuazione dei primi focolai della malattia nel lodigiano e nel padovano. A partire da tale data e parallelamente alla diffusione crescente dell'epidemia nel nostro paese, l'uso della parola inizia ad aumentare in modo esponenziale, con la pubblicazione quotidiana di numerosi articoli e interventi, che approfondiscono l'argomento citando il virus responsabile della malattia, di cui del resto si parla anche nella maggior parte dei servizi dei telegiornali e di altri programmi televisivi, oltre che sui social e nelle comuni conversazioni quotidiane tra parlanti: al 2I/3/2020 sono oltre 700 milioni le occorrenze della parola riscontrate nelle pagine italiane di Google e migliaia quelle rilevate nei soli primi tre mesi dell'anno negli archivi dei principali quotidiani nazionali (rispettivamente r.or4 nel "Corriere della Sera", 7.995 nella "Repubblica" e 5.715 nella "Stampa").

Losservazione di tali occorrenze ci rivela inoltre come al corretto uso iniziale del sostantivo coronavirus, usato come nome comune indicante la tipologia di virus (da qualificare poi attraverso il ricorso a un aggettivo o ad altre specificazioni che identifichino con precisione il particolare virus cui ci si riferisce, come in "nuovo coronavirus", o "(nuovo) coronavirus di Wuhan", o ancora "(nuovo) coronavirus cinese"), si sia presto sostituito un uso antonomastico della parola, che non indicherebbe più un qualsiasi coronavirus della famiglia, bensì il coronavirus responsabile della nuova epidemia. Nei media italiani il virus, pur designato ufficialmente dall'Oms prima come 2019-nCoV (ossia 2019 Novel Coronavirus) e poi come SARS-CoV-2 (acronimo di Severe Acute Respiratory Syndrome Coronavirus 2, per la stretta parentela con il coronavirus responsabile della SARS), viene di conseguenza più spesso indicato semplicemente come "il coronavirus" (talora anche con la lettera maiuscola), come avviene per esempio nei seguenti passi:

Secondo le autorità sanitarie cinesi il coronavirus «non è potente quanto la Sars», ma sta diventando più contagioso. (s.n., "Coronavirus meno potente, ma più contagioso della Sars". Pechino vieta il commercio degli animali selvatici. In Italia i controlli sugli aerei, laStampa.it, sez. Esteri, 26/I/2020)

Aumenta anche il numero degli operatori sanitari contagiati in Italia dal Coronavirus: mercoledi, secondo i dati dell'Istituto Superiore di Sanità, erano saliti a 6.205, vale a dire più del $9 \%$ dei casi totali. 
(Alessandro Fulloni, Coronavirus, i medici morti sono già 39. In Italia un contagiato su dieci è un sanitario, Corriere.it, sez. Cronache, 26/3/2020)

Con tale uso antonomastico, la parola ricorre anche nella variante virus corona, decisamente minoritaria (con sole II2.00o occorrenze in rete), e in quella "ridotta" corona, che risulta però più propria di un registro colloquiale ed è impiegata soprattutto in forma di hashtag sui social. Per esempio:

Ha portato una bottiglia e bicchierini separati per ciascun fronte, si stappa e si brinda a distanza. «Perché il bicchiere è sempre mezzo pieno e noi il "corona" lo battiamo così dice Simona. (Laura Gozzini, Brindisi e paste al check point, "la Repubblica", sez. Cronaca, p. 7, 8/3/2020)

Ma ho come l'idea che il virus corona ci cambierà per sempre, economicamente e socialmente, come non è riuscito al terrorismo politico, allo shock petrolifero, all'islamismo radicale, alla crisi finanziaria. Credo che il corona segnerà il nostro tempo come la spagnola o la poliomelite o la guerra hanno temprato le generazioni precedenti. (Christian Rocca, Il corona non è solo un virus, é l'inizio di una nuova èra, Linkiesta.it, $13 / 3 / 2020)$

Per estensione, il sostantivo coronavirus ha inoltre cominciato a essere impiegato anche per indicare la malattia respiratoria causata dal SARS-CoV-2, a cui l'Organizzazione mondiale della sanità l'ir febbraio del 2020 ha dato il nome ufficiale di COVID-I9 (acronimo dell'inglese CoronaVirus Disease 19 'malattia da coronavirus I9'), ma che, anche dopo tale data, nei giornali e nella lingua corrente ha continuato a essere più spesso indicata come "sindrome (o malattia, o infezione) da coronavirus", o appunto, più sinteticamente, come "coronavirus" (in molti casi anche con la lettera maiuscola):

Raffaele Morelli è uno psichiatra e psicoterapeuta. [...] Nel video che vi proponiamo afferma con assoluta pacatezza e senza isterie come si può convivere con una malattia come il coronavirus. (Francesco Storace, Mandate questo video in tv, basta con la paranoia da coronavirus che angoscia l'Italia, Secolod'Italia.it, sez. Cronaca, 5/3/2020)

Altra cosa sperimentare in diretta TV giorno per giorno i drammi attuali della nostra epoca, dai cambiamenti climatici ad una infezione come il Coronavirus che corre alla velocità della luce. (Pino Currò, Noi e il Coronavirus. Ne usciremo piu forti di prima, Tempostretto.it, 7/3/2020)

In questi ultimi due più recenti significati (in quello antonomastico di 'coronavirus SARS-CoV-2', e in quello esteso di 'malattia respiratoria causata dal SARS-CoV-2'), la forma è registrata nel portale Treccani come "Neologismo 2020", insieme al significato originario di 'genere di virus responsabili di diverse malattie nell'uomo e negli animali, prevalentemente respiratorie e polmonari', che è invece l'unico con cui la voce risulta per il momento accolta nei principali dizionari sincronici, tra cui il Garzanti, il Devoto-Oli e lo Zingarelli (questi ultimi consultati nelle edizioni 2020, edite però nel 2019, che riportano come data di prima attestazione rispettivamente il 2003 e il I990).

Infine, dal punto di vista grafico la forma più corretta della parola è quella sintetica, con grafia univerbata (che è del resto anche quella prevalente nell'uso e l'unica ad essere accolta dai lessicografi), e con la lettera iniziale minuscola (coronavirus): nel suo valore originario, il sostantivo rappresenta infatti un nome comune, che indica il genere di appartenenza del virus e non il nome proprio di uno specifico rappresentante di tale gruppo di virus, e come tale non richiede quindi l'uso della maiuscola. Il ricorso a quest'ultima, di cui si rinvengono numerose occorrenze, è probabilmente determinato dal fatto che la parola, come si è detto, viene talora impiegata con funzione di nome proprio, per indicare per antonomasia il virus responsabile del COVID-I9, o per estensione la malattia respiratoria 
provocata dal nuovo virus: neppure in tali casi l'uso della maiuscola è tuttavia giustificabile, dato che in italiano i nomi dei virus, cosi come i nomi delle malattie, si scrivono con l'iniziale minuscola (si veda in proposito la scheda sul nome ebola di Maria Cristina Torchia).

Quanto alla pronuncia del termine, la più corretta è quella italiana, coronavirus, e non quella anglicizzante "coronavairus": come osservato dal presidente dell'Accademia nel citato tema del mese, la parola, pur introdotta in italiano come prestito integrale dall'inglese, è stata infatti da subito adattata alla pronuncia della nostra lingua, anche in virtù del fatto che i due elementi componenti del composto, corona e virus, entrambi di origine latina, sono attestati anche in italiano e come tali sono di conseguenza pronunciati. Non ci sono quindi ragioni per sostituire tale pronuncia, registrata anche nei dizionari e ormai da tempo consolidata nell'uso, con quella inglese, per quanto questa non possa considerarsi propriamente scorretta.

\section{Cita come:}

Sara Giovine, Coronavirus: un nome comune (di virus) per una malattia non comune, "Italiano digitale", 2020, XII, 2020/1 (gennaio-marzo)

DOI: $10.35948 / 2532-9006 / 2020.3312$

Copyright 2020 Accademia della Crusca

Pubblicato con licenza creative commons CC BY-NC-ND 